\title{
Éditorial:
}

\section{Nouveaux modèles du vieillissement: des efforts créateurs et le besoin d'aller plus loin}

Ce numéro spécial présente de nouvelles idées sur le vieillissement qui méritent d'être connues. Les articles et les commentaires sont rédigés par des chercheurs et des universitaires émérites qui s'intéressent à la compréhension du phénomène du vieillissement. Ce matériel a été préparé à la lumière d'un nouveau point de vue selon lequel l'étude du vieillissement est devenue un domaine de connaissances riche en données mais pauvre en théorie, c'est-à-dire une collection de renseignements divers et disjoints de grande dimension. Les explications simplistes, univariées, du vieillissement ont été remplacées par la microgérontologie dominée par l'exploration d'aspects pointus du vieillissement et par la microthéorie. En réponse à cet état de fait, un symposium, intitulé Modèles du vieillissement, a été organisé en vue du Congrès international de gérontologie, tenu à Budapest au mois de juillet 1993. À cette occasion, quatre points de vue différents sur le vieillissement ont été sollicités: biologique, psychologique, social et épidémiologique-écologique.

Les conférenciers du symposium devaient discuter des modèles du vieillissement dans leur discipline, souligner leurs préférences et, s'ils le désiraient, présenter leurs propres efforts créateurs dans le but de contribuer à l'affinement de nos modèles sur le vieillissement. Leurs travaux ont été révisés et sont maintenant publiés dans le présent numéro de La Revue canadienne du vieillissement; ils sont accompagnés de commentaires et de répliques.

Ce numéro spécial est consacré à la recherche de concepts et de modèles plus efficaces du vieillissement. Les articles visent à nous aider à concevoir et mener des recherches plus adéquates, afin de mieux comprendre les processus du vieillissement et, par conséquent, jeter les assises nécessaires à l'amélioration des conditions qui mènent à la détérioration.

Selon moi, les articles et les commentaires sont des plus intéressants et j'espère qu'un grand nombre d'entre vous les lirez. J'espère également qu'ils encourageront les chercheurs à examiner sérieusement les modèles implicites et explicites dont ils se servent pour expliquer le vieillissement. J'estime qu'il m'est inutile de commenter en détail ce numéro, mais je tiens tout de même à souligner quelques points.

Par le passé, nous étions portés à emprunter nos concepts et nos modèles à nos disciplines respectives, lesquelles se souciaient peu d'expliquer le changement. À leurs débuts, les domaines de la biologie, de la psychologie et de la sociologie se fondaient sur des propriétés statiques. Nos connaissances scientifiques nous ont peu aidés à expliquer comment les cellules se différen- 
cient et subissent le phénomène de sénescence, comment une personne n'est pas uniquement le produit d'une structure sociale mais en détermine ellemême la structure, et comment le milieu et l'apprentissage modifient l'expression du génome. De toute évidence, nous devons admettre que des forces interactives existent dans le processus du vieillissement, par exemple: les grandes associations entre la morbidité et la mortalité et les classes sociales. Toutefois, ces articles n'ont pas comme objectif d'élucider le mystère de ce qui constitue les processus dynamiques. Les structures sociales et les économies ne sont pas vivantes, mais les organismes le sont. Les organismes consistent en des systèmes énergétiques de structuration autonome, contrairement aux milieux.

De la lecture de ces articles, j'ai conclu que nous avons été limités dans la conception de modèles du vieillissement, car les résultats du vieillissement se présentent comme un ensemble complexe d'interactions entre des organismes de structuration autonome et leur milieu. Les modèles du vieillissement semblent nécessiter des développements conceptuels tant sur l'énergie que sur l'information. Dans ces articles, nous relevons des termes tels entropie, négentropie et gérodynamique. Ceux-ci semblent cerner les forces du vieillissement dans des processus biologiques. Toutefois, je ne peux classer facilement toutes les variances uniquement dans la catégorie biologique, à la lumière du fait qu'au cours du siècle présent, environ 30 années ont été ajoutées à l'espérance de vie moyenne ou que les autochtones originaires de l'Arctique s'établissant en milieux urbains présentent des changements au niveau des signes physiologiqes du vieillissement.

À la suite de la lecture de ces articles, je suis porté à croire que le vieillissement consiste en un processus écologique, soit en une interaction entre des organismes à composition génétique particulière et des milieux sociaux et physiques. Selon moi, la recherche de ces interactions nous permettra de déterminer des sous-modèles du vieillissement, ou des complexes causals effectifs, terme utilisé par un des auteurs.

L'utilisation d'une seul modèle de vieillissement, qu'il soit biologique, psychologique ou social, peut être mise en doute en raison des discontinuités mentionnées dans certains de ces articles. Cependant, bon nombre d'aspects des systèmes de vie révèlent une activité rythmique. La continuité et la discontinuité, la stabilité et le changement, la différenciation et dédifférenciation font partie des termes qui cadrent avec certaines caractéristiques du vieillissement. Ces articles renferment également des termes moins connus qui remplaceront peut-être de plus vieilles expressions lorsque nous aurons amélioré notre terminologie et saisi l'organisation du vieillissement comme phénomène naturel.

Les échanges entre les auteurs et les commentateurs suggèrent que les améliorations apportées à nos modèles et théories dépendent des conclusions que nous tirerons de nos données et des méthodes que nous utiliserons pour y arriver. Des études longitudinales exhaustives et séquentielles nous aideront à classer les influences sur le vieillissement en catégories d'effets plus précises, qui résultent de changements séculaires au sein de la po- 
pulation et des différences entre groupes, du moment de la saisie des mesures, et des propriétés des organismes. Nous pourrions notamment partitionner la variance du vieillissement non seulement en fonction des caractéristiques socio-structurelles et génétiques, mais également en fonction des effets de la condition de la vie foetale sur l'âge adulte. Par exemple, les nouveaux-nés de petit poids doivent surmonter plus d'obstacles pour survivre et leur mère sont souvent célibataires et démunies sur le plan socioéconomique. Ces conditions au cours des toutes premières années de la vie ont-elles une incidence à un âge plus avancé?

La compréhension du vieillissement est une tâche très complexe. Les processus du vieillissement délimitent des domaines des plus compliqués et représentent un défi de taille pour l'étude et l'explication scientifique. C'est peut-être même la raison pour laquelle nous sommes portés à étudier certains aspects précis du vieillissement chez l'être humain et à élaborer des micro-théories. À la lumière de ces faits, ces articles ont pour but de susciter une pensée axée davantage sur l'intégration des perspectives, de lier les aspects du vieillissement dans des ensembles théoriques et explicatives plus globaux.

Notre domaine d'étude s'est éloigné des premières tentatives d'explication du vieillissement au moyen d'une variable simple, des points de vue qui voulaient qu'un certain nombre de battements de coeur nous étaient assignés, ou que le vieillissement résultait d'une putréfaction gastro-intestinale ou de l'action d'une seule hormone contrôlant la durée de notre vie. En rejetant les solutions simplistes, nous nous sommes butés aux explications plus complexes. Une personne raisonnable aurait probablement tendance à croire que le processus du vieillissement chez l'humain résulte du génome qui interagit avec les circonstances des milieux physique et social. L'accroissement marqué de l'espérance de vie au cours du siècle actuel, non seulement à la naissance mais également à un âge avancé, suggère que le vieillissement est un exemple de relations écologiques dont le résultat se modifie en fonction des nouvelles circonstances qui surgissent.

Les articles ne sont pas présentés dans un ordre particulier. Je vous suggère de lire premièrement les sujets qui se rapportent à vos intérêts, puis de passer aux autres. Les articles sont le fruit d'efforts systématiques déployés par des savants réputés dont l'objectif consiste à étudier les questions complexes et confuses du vieillissement. Lorsque vous aurez terminé votre lecture, posez-vous la question suivante: «Quel est mon modèle du vieillissement?» J'estime que cette série d'articles nous aideront à affiner les modèles du vieillissement que nous utilisons pour planifier nos recherches, pour enseigner la gérontologie et organiser des services à l'intention des personnes âgées et de notre société vieillissante.

Je suis fier d'avoir eu l'occasion d'organiser le symposium sur les modèles du vieillissement qui a permis la publication du présent numéro. Je suis reconnaissant des efforts des participants et des commentaires des réviseurs. Monsieur François Béland, le rédacteur en chef de la Revue, doit être remercié de l'intérêt et de l'appui dont il a fait preuve envers la réalisation de cette 
publication. Ce numéro spécial de la Revue sera lu dans un grand nombre de pays par des universitaires chevronnés, des étudiants et des professionnels qui désirent comprendre le vieillissement, c'est-à-dire l'un des aspects les moins compris de notre existence.

J'espère que les lecteurs se joindront à moi pour remercier les auteurs de leur travail, les commentateurs de leurs remarques réfléchies, ainsi que les auteurs de leur réplique. Ce numéro présente de solides réflexions, mais également des critiques frappantes, soient les éléments idéaux pour créer une tribune scientifique productive. Monsieur François Béland nous a rendu un immense service en encourageant la publication de cet effort de consolidation de nos pensées sur une question très complexe et importante: le vieillissement.

James E. Birren 